Ti me- peri odi c magnet i c fi el d anal ysi s with sat ur at i on and hyst er esi s char acteri sti cs by har moni $c$ bal ance fi ni te el ement met hod

\begin{tabular}{|l|l|}
\hline 著者 & Yamada Sot oshi, Lu Junwei, Bessho Kazuo \\
\hline $\begin{array}{l}\text { j our nal or } \\
\text { publ i cat i on t i t l e }\end{array}$ & I EEE Transact i ons on Nagget i cs \\
\hline vol ume & 26 \\
\hline nunber & 2 \\
\hline page r ange & $995-998$ \\
\hline year & $1990-04-01$ \\
\hline URL & ht t p: //hdl . handl e. net /2297/48291 \\
\hline
\end{tabular}




\title{
TIME-PERIODIC MAGNETIC FIELD ANALYSIS WITH SATURATION AND HYSTERESIS CHARACTERISTICS BY HARMONIC BALANCE FINITE ELEMENT METHOD
}

\author{
J.Lu S.Yamada K.Bessho \\ Electrical Energy Conversion Lab. \\ Faculty of Technology \\ Kanazawa University \\ Kanazawa, 920, Japan
}

\begin{abstract}
In this paper we shall further discuss the harmonlc balance finite element method (HBFEM) for the time-periodic magnetic field with saturation and hysteresis characteristics and 1ts applications. The HBFEM enables us to calculate the distribution of harmonic magnetic flux at an $A C$ magnetization, and it dose not need the intricate calculation concerned with the time variation. Comparisons between. numerical analysis and experimental results are presented.
\end{abstract}

\section{Introduction}

The HBFEM is a new method which deals with time-perlodic magnetic fleld problems. This HBFEM is combined by FEM and harmonic balance method (1), and developed for the purpose of analyzing the time-periodic problems with a saturated core. The distribution of magnetic flux for each harmonic is provided by HBFEM. Since the HBFEM works in the harmonic domain, we do not need to calculate the time variation directly.

In this paper the formulation of the HBFEM is described for the 2-dimensional time-periodic corresponding to any expression of the magnetizing characteristics including the hysteresis, and the comparisons are made between numerical analysis and experimental results. The formulation for the magnetizing characteristics expressed by low-order polynomial functions is discussed. The HBFEM is also suited to an $A C$ magnetic field connected with voltage source and the external circult. Several examples are given in this paper.

\section{Formulation of HBFEM}

As mentioned in the above section, the HBFEM is a new method which is combined by FEM and Harmonic Balance. For simplicity of the formulation, a magnetic fleld is assumed as two-dimensional in the $(x, y)$ plane, the present problem is quasi-stationary. Therefore, the vector potential $A(0,0, A)$ satisfles the region of interest surrounded with some boundary conditions

$$
\frac{\partial}{\partial x}\left(v \frac{\partial A}{\partial x}\right)+\frac{\partial}{\partial y}\left(v \frac{\partial A}{\partial y}\right)=-J \cdot+\sigma \frac{\partial A}{\partial t}
$$

where $\gamma$ and $\sigma$ are the magnetic reluctivity and the conductivity. The formulation $1 \mathrm{~s}$ made by use of the Galerkin procedure. Its integral form is

$$
\begin{gathered}
\int_{Q} J\left\{\frac{\partial N_{1}}{\partial x}\left(v \frac{\partial A}{\partial x}\right)+\frac{\partial N_{1}}{\partial y}\left(v \frac{\partial A}{\partial y}\right)\right\} d x d y \\
-\int_{Q} J\left(J--\sigma \frac{\partial A}{\partial t}\right) N_{1} d x d y=0
\end{gathered}
$$

where $N_{i}(x, y)$ is the interpolating function. We are only interested in the timeperiodic solution (the harmontc problem), when an alternating magnetizing current is applied. According to the harmonic balance method all variables, such as vector potentials A, flux densities $B$ and magnetizing current $J$, are approximated as harmonic solutions, the expression of function is

$$
\begin{aligned}
& A^{\prime}=\sum\left\{A_{n}{ }^{\prime} \sin (n \omega t)+A_{n \cdot} \cdot \cos (n \omega t)\right\} \\
& n=1,1.1 . .1 \\
& B x^{*}=\sum\left\{B x_{n+} \cdot \sin (n \omega t)+B x_{n 0^{\circ}} \cos (n \omega t)\right\} \\
& n=1.3 .8 \ldots \\
& B y^{*}=\Sigma\left\{B y_{n} 0^{*} \sin (n \omega t)+B y_{n 0^{\circ}} \cos (n \omega t)\right\} \\
& n=1.3 .8 \ldots \\
& J_{0}{ }^{i}=\Sigma\left(J_{n},^{\prime} \sin (n \omega t)+J_{n o} \cos (n \omega t)\right\}
\end{aligned}
$$

where $\omega$ is the fundamental angular frequency. The magnetizing characteristic of the core can be expressed by an arbitrary function of the flux density $B$, that is

$$
H(B)=H_{n A}(B)+H \ldots n(B) \frac{d B}{d t}
$$

where the first term is saturation characteristic, and the second term indicates the hysteresis characteristics (2). The magnetic reluctivity which is obtalned by the Fourier expansion has the form of

$$
\begin{aligned}
v(t) & =H\{B(t)\} / B(t) \\
& =v_{0}+\sum\left\{v_{n=2}, \sin (n \omega t)+v_{n} \cos (n \omega t)\right\}
\end{aligned}
$$

where

$$
\begin{aligned}
& B=\left(B v^{2}+B v^{2}\right) 1 / 2 \\
& v_{n}=1 / T \int^{1} v(t) d t \\
& v_{n}=2 / T \int^{\prime} v(t) \sin (n \omega t) d t \\
& v_{n t}=2 / T \int^{T} v(t) \cos (\pi \omega t) d t
\end{aligned}
$$

Substituting Eqs. (3) and (5) into Eq. (2) and equating the coefficlents of sin (nwt) and $\cos (n \omega t)(n=1,3, \ldots)$ on both sides according to the harmonic balance method, as a result, the matrix for one element is obtalned as follows :

$$
\begin{aligned}
& \frac{1}{4 \Delta}\left[\begin{array}{ccc}
\left(b_{1} b_{1}+c_{1} c_{1}\right) D & \left(b_{1} b_{2}+c_{1} c_{2}\right) D & \left(b_{1} b_{3}+c_{1} c_{3}\right) D \\
\left(b_{2} b_{1}+c_{2} c_{1}\right) D & \left(b_{2} b_{2}+c_{2} c_{2}\right) D & \left(b_{2} b_{3}+c_{2} c_{2}\right) D \\
\left(b_{3} b_{1}+c_{3} c_{1}\right) D & \left(b_{1} b_{2}+c_{3} c_{2}\right) D & \left(b_{3} b_{2}+c_{3} c_{3}\right) D
\end{array}\right]\{A \cdot\} \\
& +\frac{\sigma \omega \Delta}{12}\left[\begin{array}{ccc}
2 N & N & N \\
N & 2 N & N \\
N & N & 2 N
\end{array}\right]\{A \cdot\}-.\{K \cdot\}
\end{aligned}
$$

where

$$
\begin{aligned}
& b_{i}=y_{1}-y_{k}, c_{1}=x_{k}-x_{1}, \Delta: \text { cross section } \\
& \left\{A^{\circ}\right\}=\left\{A_{1},^{\prime} A_{10} 0^{\prime} A_{3,} A_{3} A^{\prime}, \ldots\right. \\
& A_{1},{ }^{2} A_{10}{ }^{2} A_{12}{ }^{2} A_{10}{ }^{2}, \cdots \text {, } \\
& \left.A_{1},{ }^{3} A_{18}, A_{3}, A_{30}, \ldots,\right\}
\end{aligned}
$$




$$
\{\mathrm{K} \cdot\}=\Delta / 3\left\{\begin{array}{llll}
\mathrm{J}_{1}, & J_{10} & J_{3}, & J_{30}, \cdots, \\
J_{1}, & J_{10} & J_{3}, & J_{30}, \cdots, \\
J_{1}, J_{10} & \left.J_{3}, J_{30}, \cdots,\right\}
\end{array}\right.
$$

The coefficients of the block matrix $D$ are determined by only the Fourier coefficlents in Eq. (7). The matr $1 \times D$ acts as a reluctivity and is called RELUCTIVITY MATRIX. On the other hand, the matrix $\mathbf{N}$ is a constant concerned with harmonic orders and is called HARMONIC MATRIX. The block matrices $D$ and $\mathrm{N}$ are given in ( 3 ).

The system equation for the entire region is obtained by the same procedure as the conventional FEM, and is solved by the iteration procedure for a nonlinear static magnetic field. When the harmonic components is up to $(2 \mathrm{~m}-1)$ order, the size of the block matrices $D$ and $N$ become $2 m \times 2 m$ and the order of the system matrix is $2 \mathrm{~m}$ times bigger than the number of nodes. But it is a sparse and band matrix.

\section{Applications of HBFEM}

To 1llustrate the application of this method, several samples are given as the analysis models in which the eddy-current, saturation and hysteresis characteristics are considered.

\section{Analysis with Eddy-current}

Usually the HBFEM is used for the timeperiodic magnetic field problems considered with non-linear magnetizing characteristic. The analysis model is given in Fig.1-a, where the shading coll and air gap are applied. Eddy-currents are Induced in the shading col1. The magnetic core $1 \mathrm{~s} \mathrm{Mn}-\mathrm{zn}$ ferrite and its magnetizing characteristic is obtained approximately as follows:

| B | $<0.400: \| 1=90.5 B+1.58 \times 10^{3} B^{3}$

$0.400<|B|<0.435: H= \pm 50.0+1.43 \times 10^{3}(8 \mp 0.400)$

$0.435<|B|<0.460: H= \pm 100 .+4.00 \times 10^{7}(B \mp 0.435)$

$0.460<18 \mid<0.490: H= \pm 200 .+1.00 \times 10^{4}(B \mp 0.460)$

$0.490<\mid$ | $\mid<0.510: \|= \pm 500 .+2.00 \times 10^{1}(\theta \mp 0.490)$

$0.510<|B|<0.520: \|= \pm 900 .+6.00 \times 10^{4}(B \mp 0.510)$

$0.520<|B| \quad: \|= \pm 1.500 .+1.00 \times 10^{3}(B \mp 0.520)$

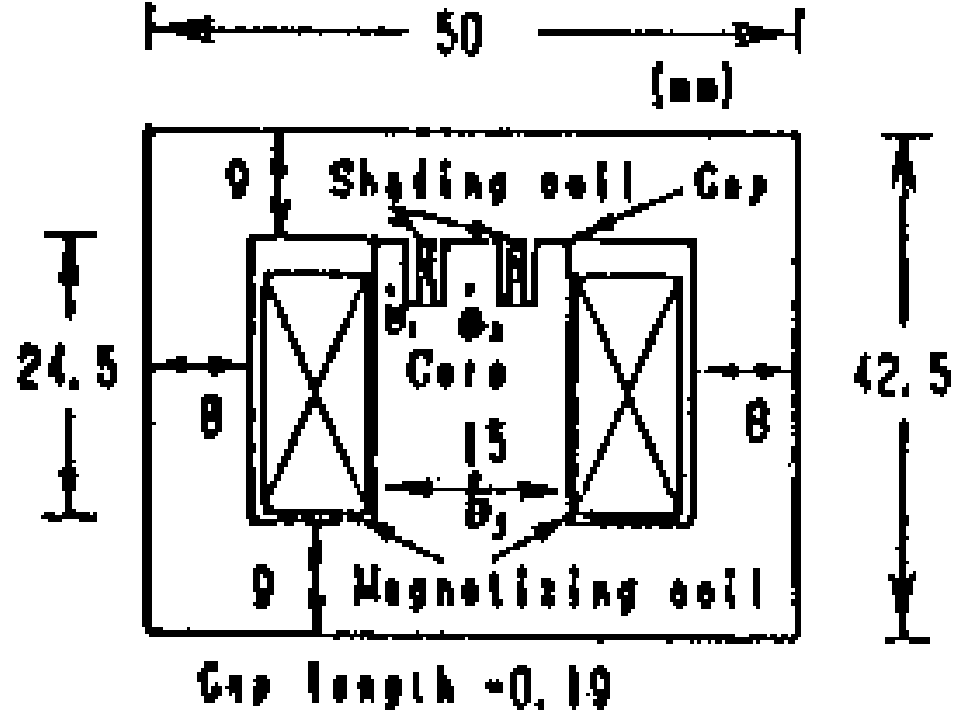

$1-a$

Fig.1 Analysis model and approximated magnetizing curve

The analysis region is a half of the cross section. The magnetizing characteristic is shown in Fig.1-b. The number of nodes and triangle element are 195 and 336 respectively. The parameters for the calculation are given as follows:

$$
\begin{array}{ll}
J_{1 s}=1.0 \times 10^{\circ} \mathrm{A} / \mathrm{m}^{2} & \sigma=3.551 \times 10^{7} \mathrm{~s} / \mathrm{m} \\
J_{3 s}=-5.4 \times 10^{4} \mathrm{~A} / \mathrm{m}^{2} & f=18011 \mathrm{z} . \\
J_{1 \mathrm{c}}=J_{\mathrm{sc}}=J_{\mathrm{sc}}=J_{\mathrm{s}}=0 &
\end{array}
$$

In this problem, the fundamental, third and fifth order harmonics are considered in the numerical analysis. The equi-potential lines of the harmonics are drawn at phase $t=0,60,90$ respectively, as shown in Fig.2, where the values of the third and fifth harmontcs are enlarged by 2.5 and 10 times respectively. In this case the numerical error is appeared, because the third and fifth harmonics are too small.

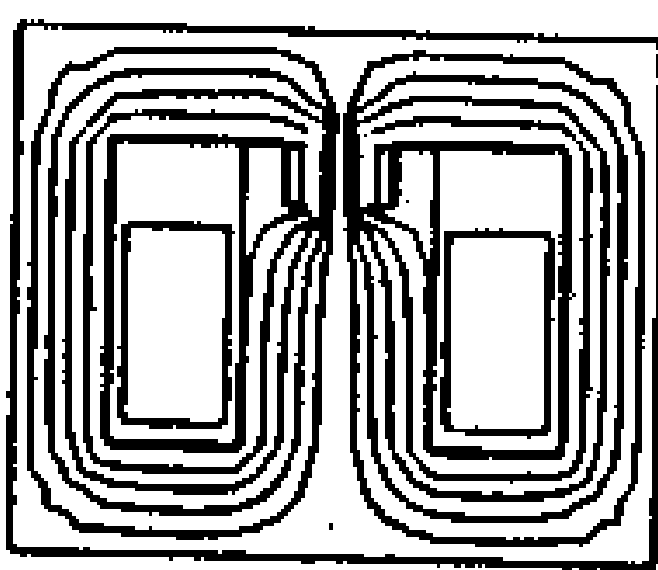

$\omega t=0$

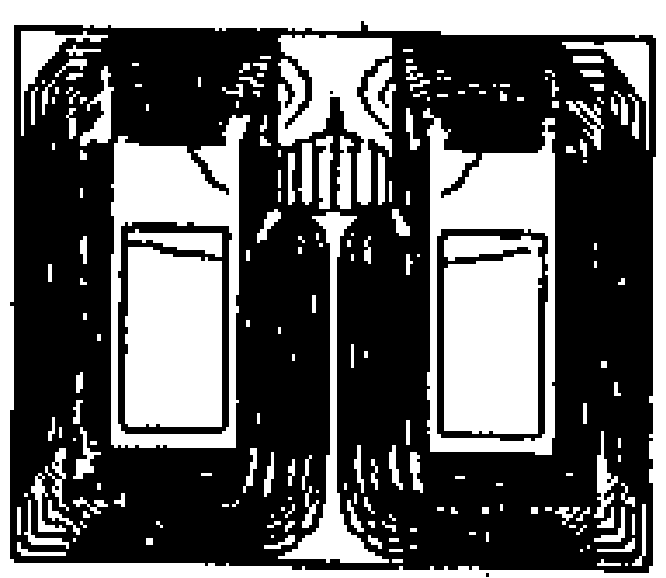

$\omega t=60$

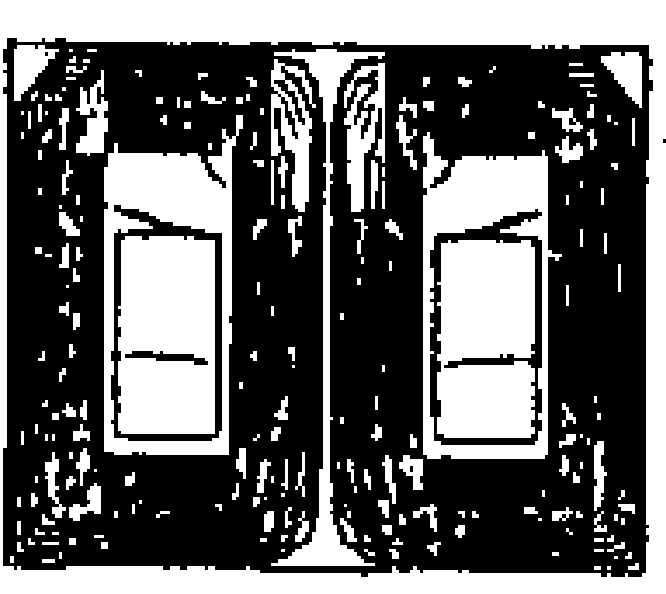

$\omega t=9 \dot{0}$

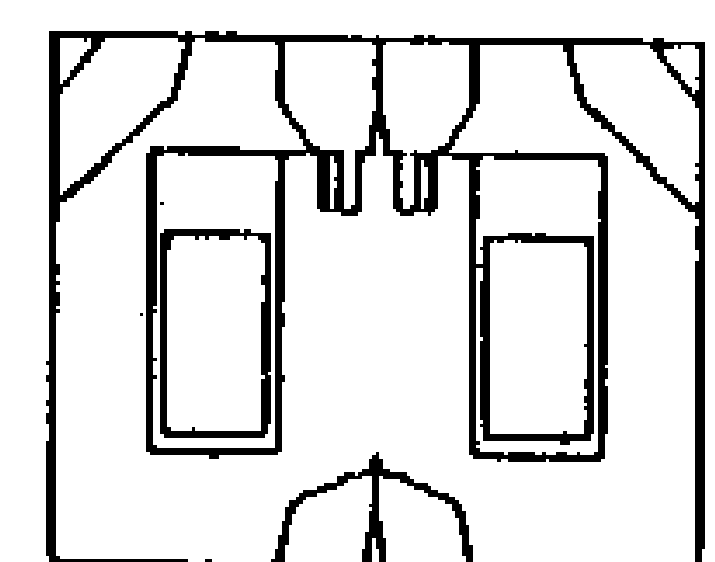

$3 \omega t=0$

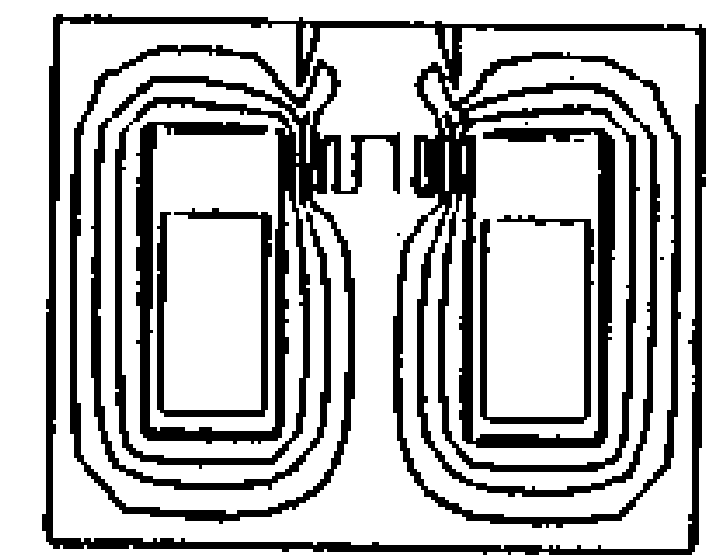

$30 \mathrm{t}=60$

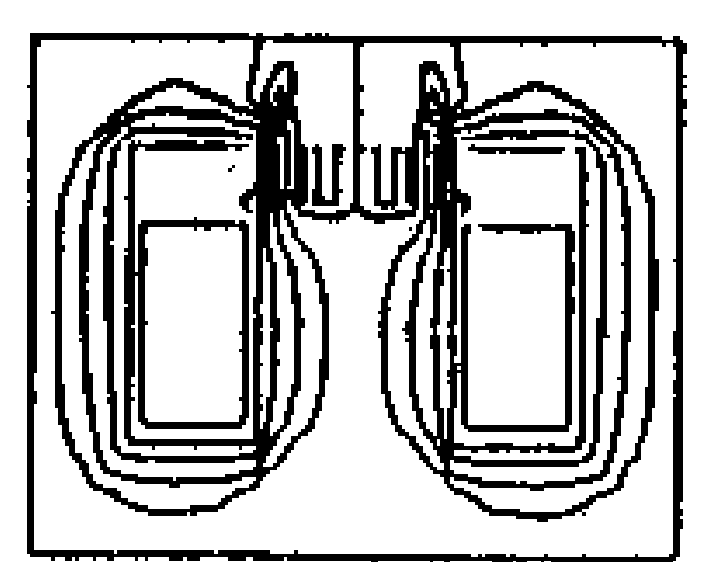

$3 \omega t=90$

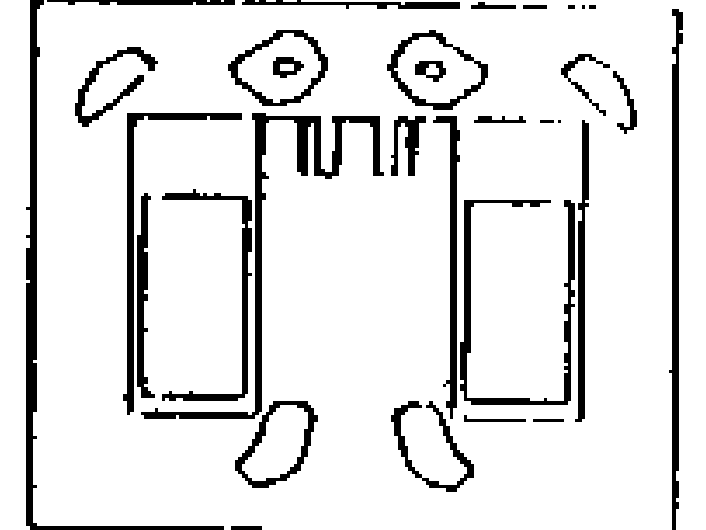

5 ov $t=0$

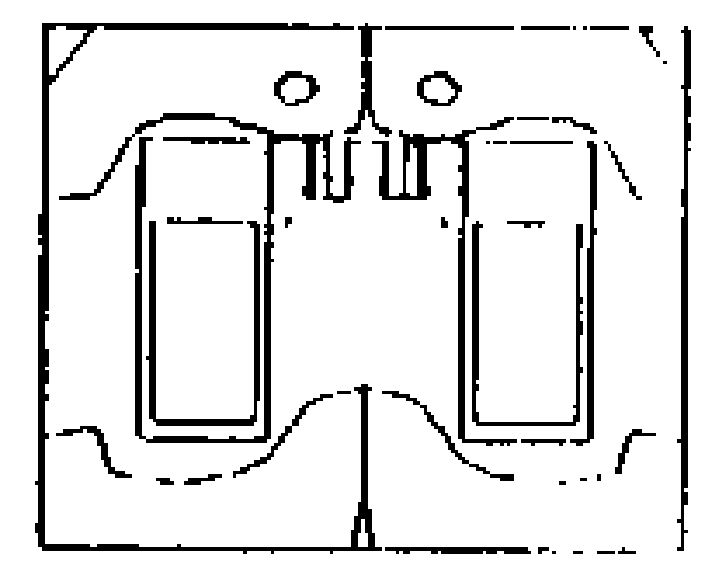

$5 \omega t=60$

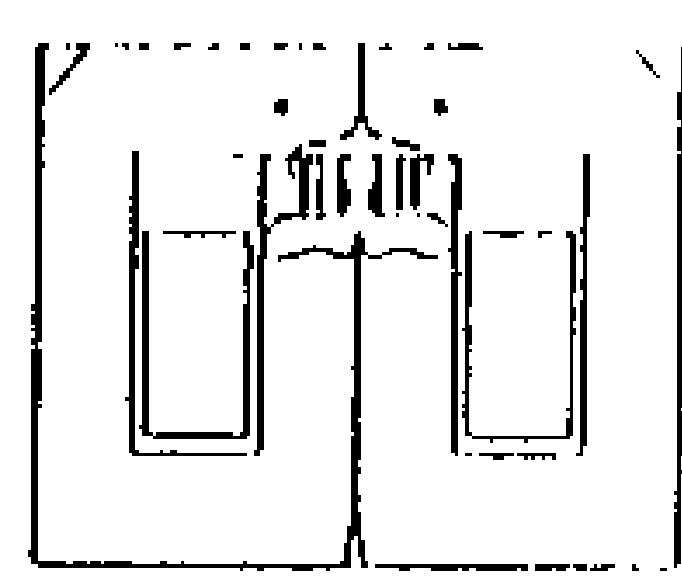

$50 t=90$
Fig.2 Distribution of Flux at $180 \mathrm{~Hz}$

F1g. 3 shows the waveform of the flux density and the magnetizing current, and the comparisons are made between numerical analysis and experimental results. The numerical analysis shows a reasonable agreement. In $\mathrm{fig.1,} B 1$ and $\mathrm{B} 2$ indicate the flux denstties inside and outside the shading coll. B3 is the flux density in the middle of leg. The magnetic flux is delayed inside the shading coil and the waveform is sinusoldal. The flux density outside the shading coil turns to the saturating state, and then the third harmonic component is induced.
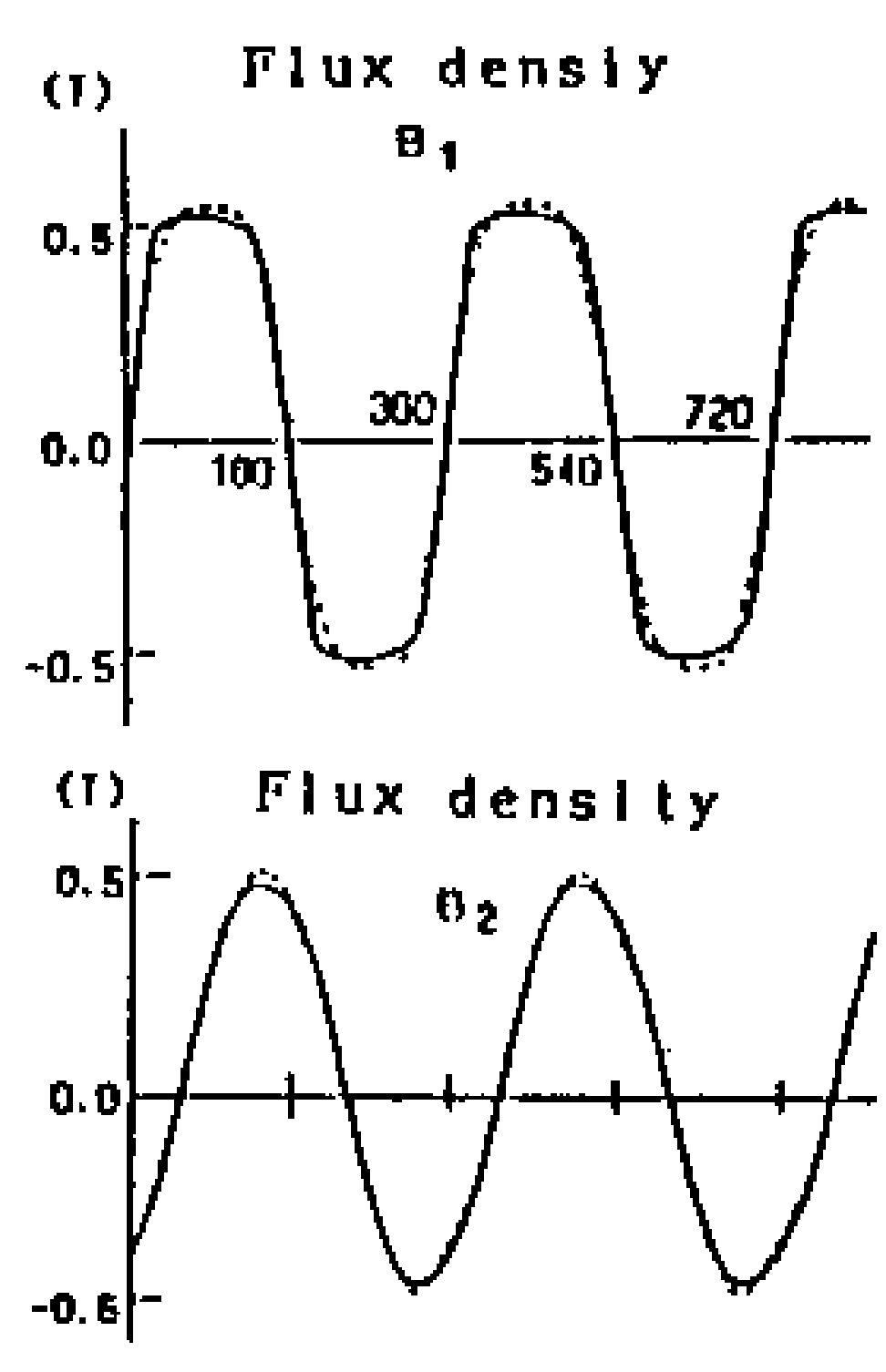

... analysis results experimental results

Fig. 3 Waveform of flux density

and magnetizing current

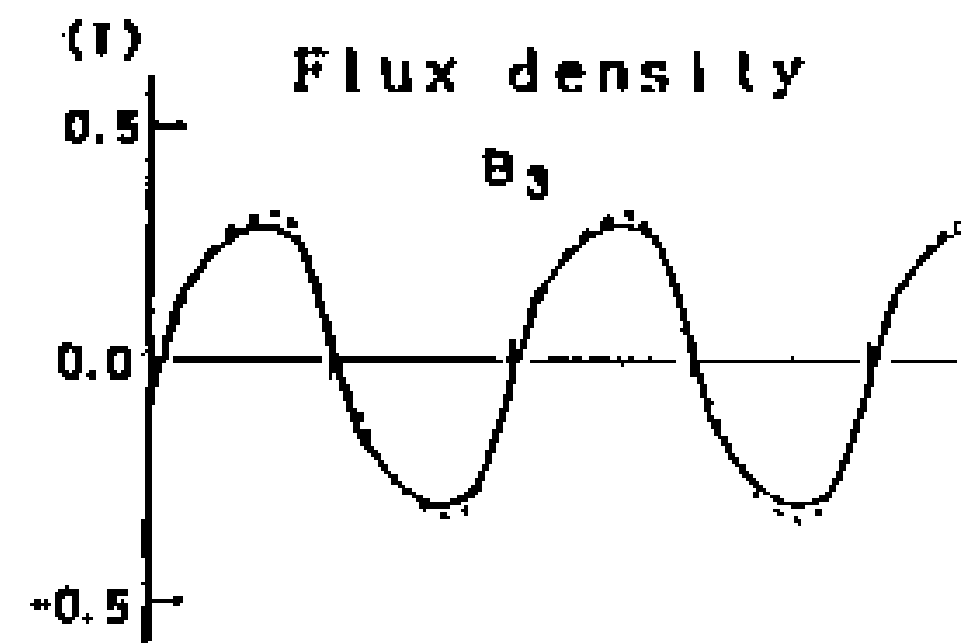

Magnetlzing current 0 ( $\left(1 / m^{2}\right)$ densl ly

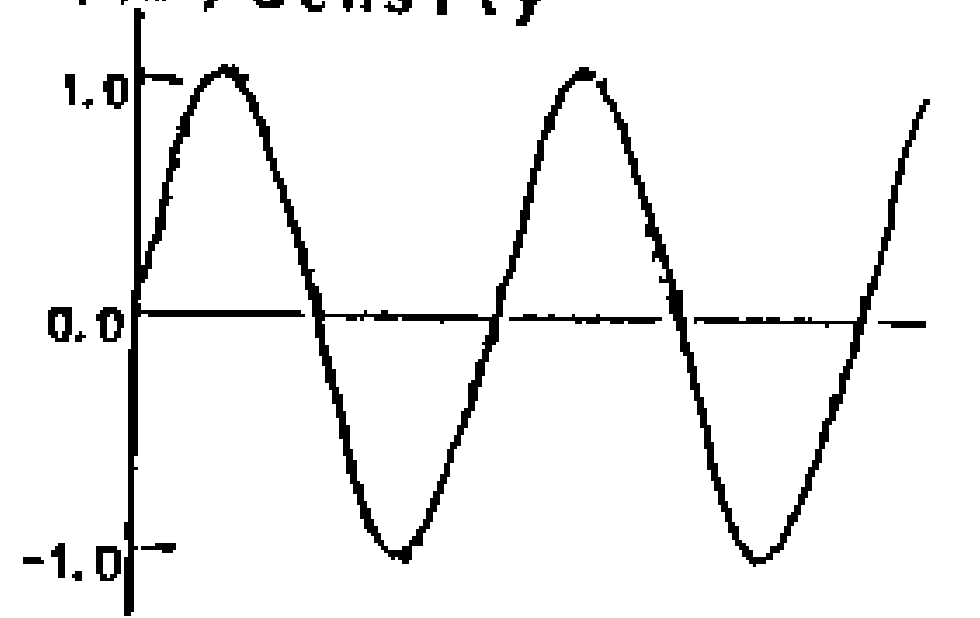


Analysis with Voltage Sources and

\section{External Circuits}

Magnetic field is usually connected to the voltage sources and the external circuit which contains resistance, inductance and capacitance. The HBFEM is also suitable to solve such kind of problems. For instance, a typical magnetic frequency tripler is shown in Fig.4, which contains three-phase commerclal power sources as an input, singlephase with 3 times commercial frequency as an output and external circuit connected to magnetic field.

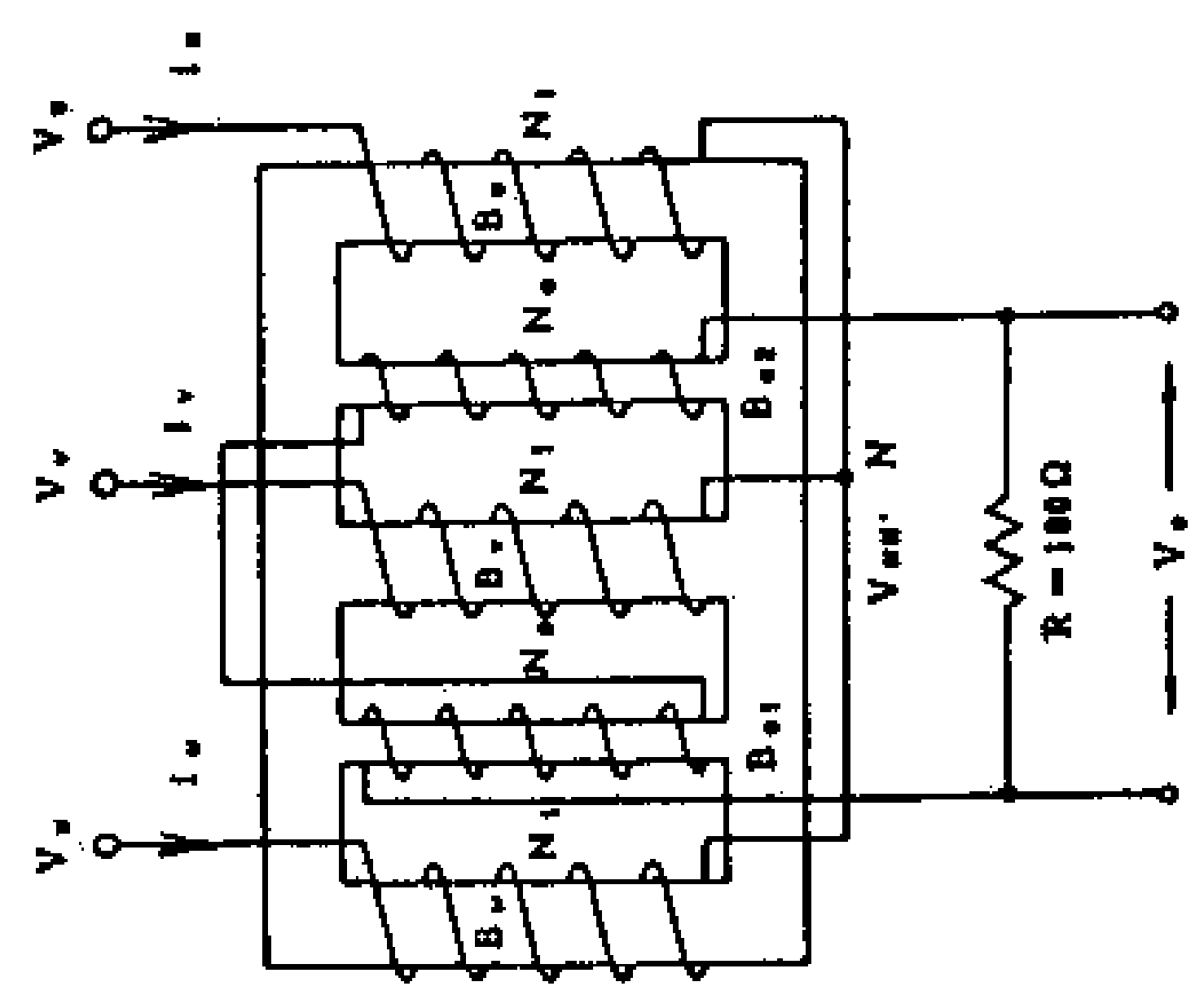

Fig. 4 Magnetic frequency tripler

To this problem an approximated magnetizing characteristic is given as follows:

$$
\mathrm{H}=100 \mathrm{~B}+40.4 \mathrm{~B}^{\circ}
$$

Based on Faraday's and Kirchhoff's laws, the matrix equation for this problem can be obtained as follows:

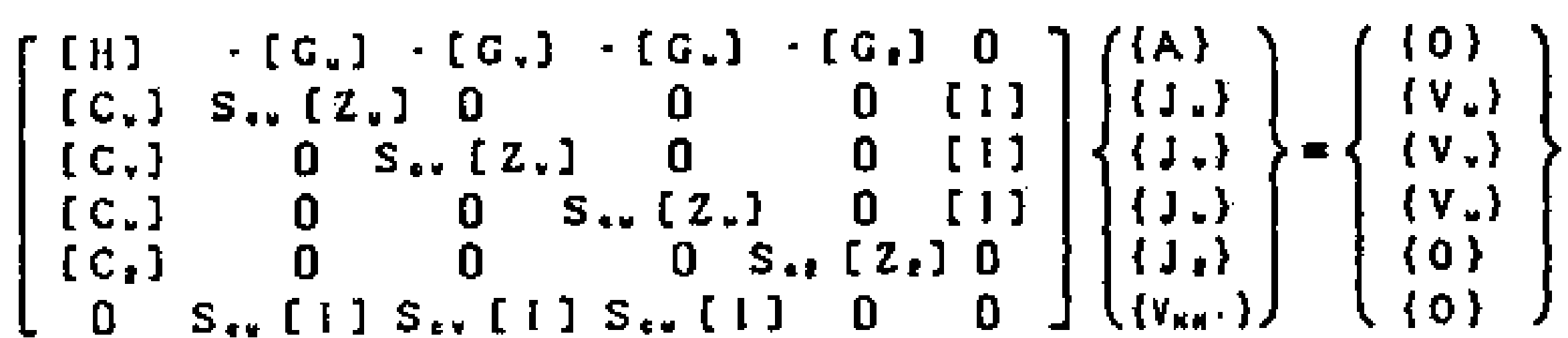

where $S_{x},[2]$ and $[I]$ are the area of each coil, the impedance matrix of circuit and unit matrix respectively.

$$
\begin{aligned}
& \left\{v_{4}\right\}=\left\{v_{41}, \quad v_{010} \quad v_{03}, \quad v_{420}, \cdots\right\} \\
& \left\{V_{v}\right\}=\left\{V_{v 11} \quad V_{v 10} \quad V_{v 3} \quad V_{n 30} \ldots\right\} \\
& \left\{V_{0}\right\}=\left\{V_{41}, \quad V_{n 10} \quad V_{n+1} \quad V_{030} \cdots\right\}
\end{aligned}
$$

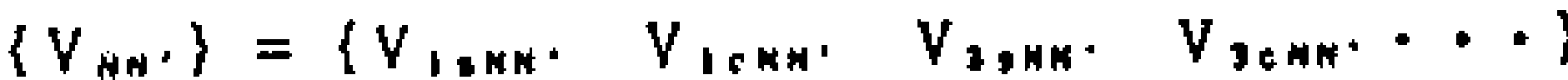

$$
\left[c_{k}\right]=\frac{\omega d_{0} \Delta}{3 S_{0 k}}[N N N]
$$

where do is the depth of model in $z$ direction.

$$
\left[G_{k}\right]=\Delta / 3
$$

We use $1 / 2$ cross section of magnetic field for analyzed region, where the number of nodes and triangle element are 340 and 594 respectively. The results of the waveform of voltage, current and flux density are given in Fig. 5.

The distribution of flux in the core is shown in Fig.6, where the distribution of third harmonic flux can be clearly observed.
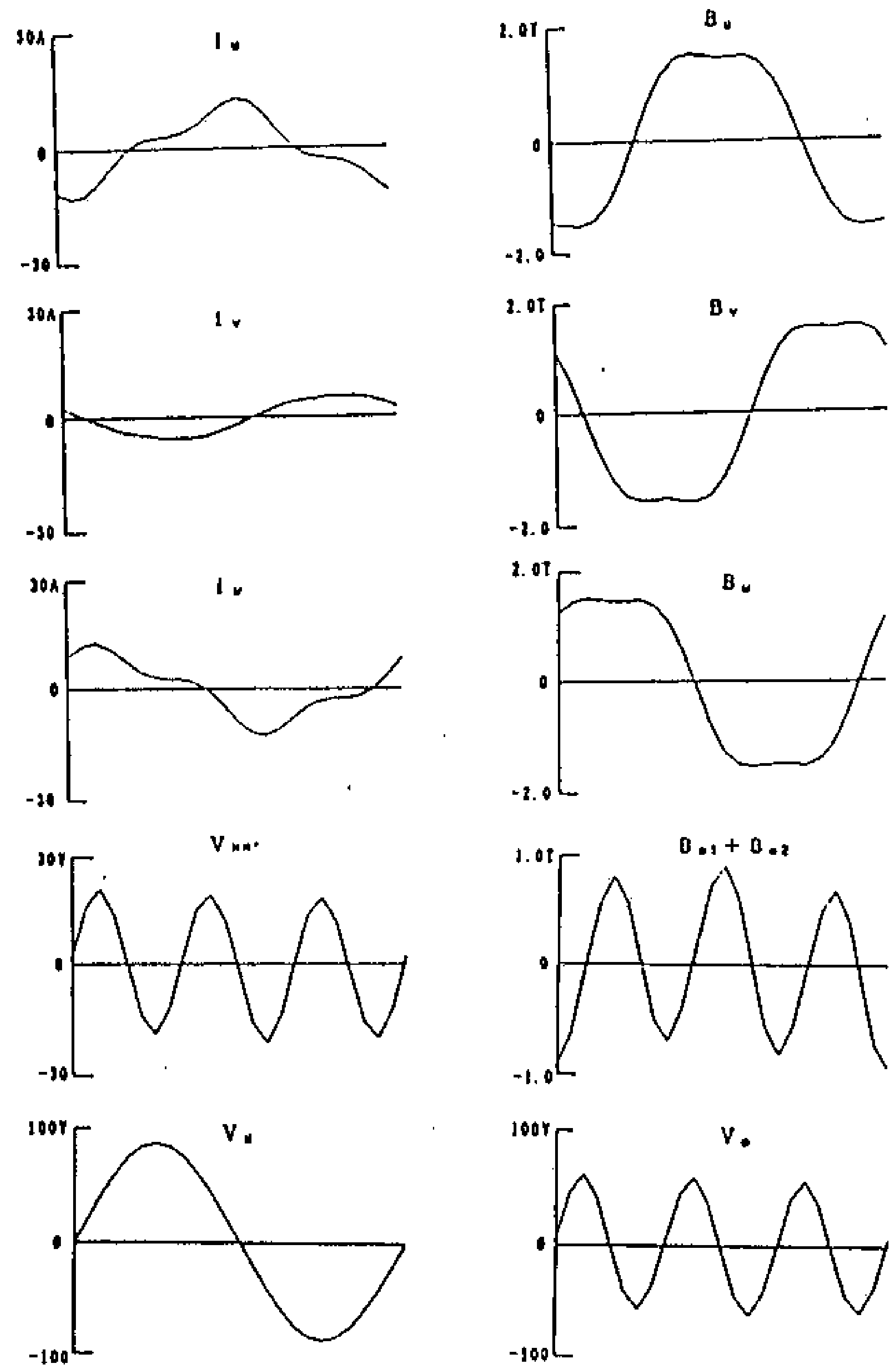

Fig.5 Results of numerical analysis
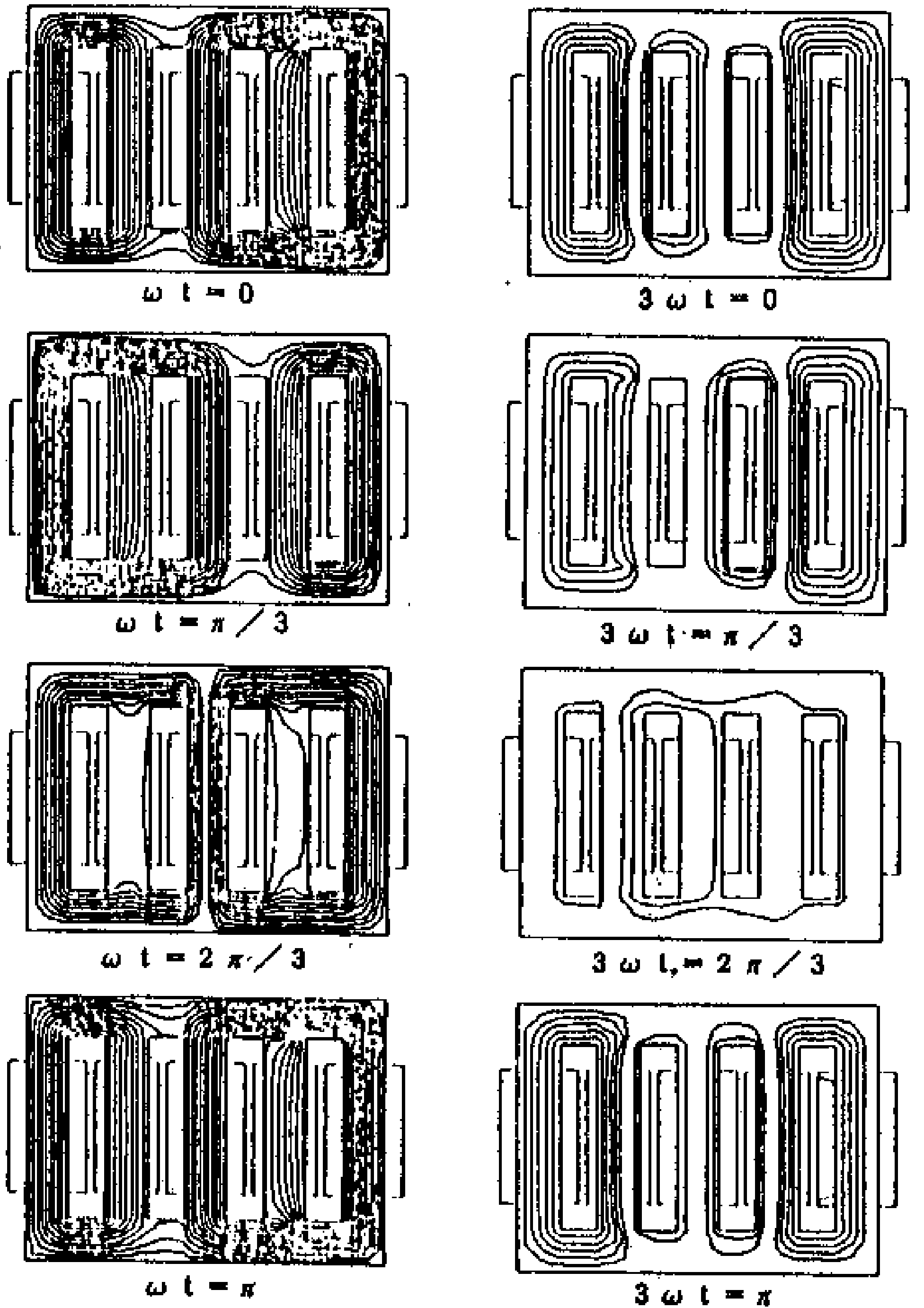

Fig.6 Distribution of flux 


\section{Analysis with Hysteresis Characteristic}

A simple Magnetic reactor is used for an analysis model as shown in Fig.7. The magnetizing curve with hysteresis characteristics is expressed as shown in Fig.8. As the calculation of time component is not considered, the flux distribution for each harmonic component can be directly obtained by HBFEM. Evidently, it is convenient to solve such problems whlch the effect of hysterests is considered. Fig.9 shows the flux distributions to each harmonic component. The waveform of flux density near the $T$-connection of the core is shown in Fig.10. The rotating flelds are observed in the corner of the core, as shown in Fig. 11 .

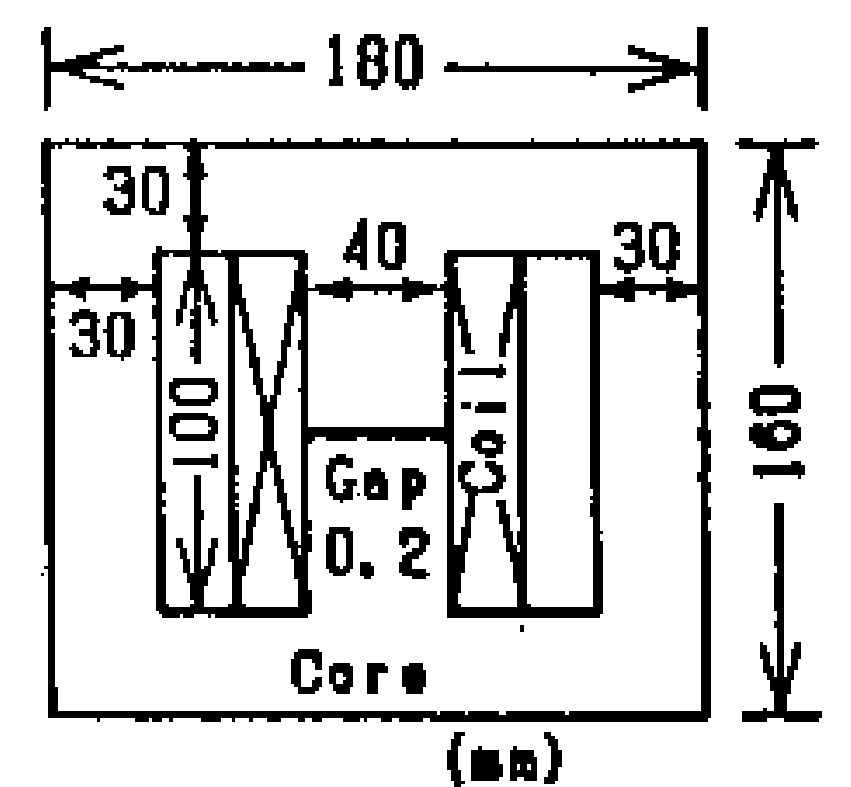

Fig.7 Analysis model

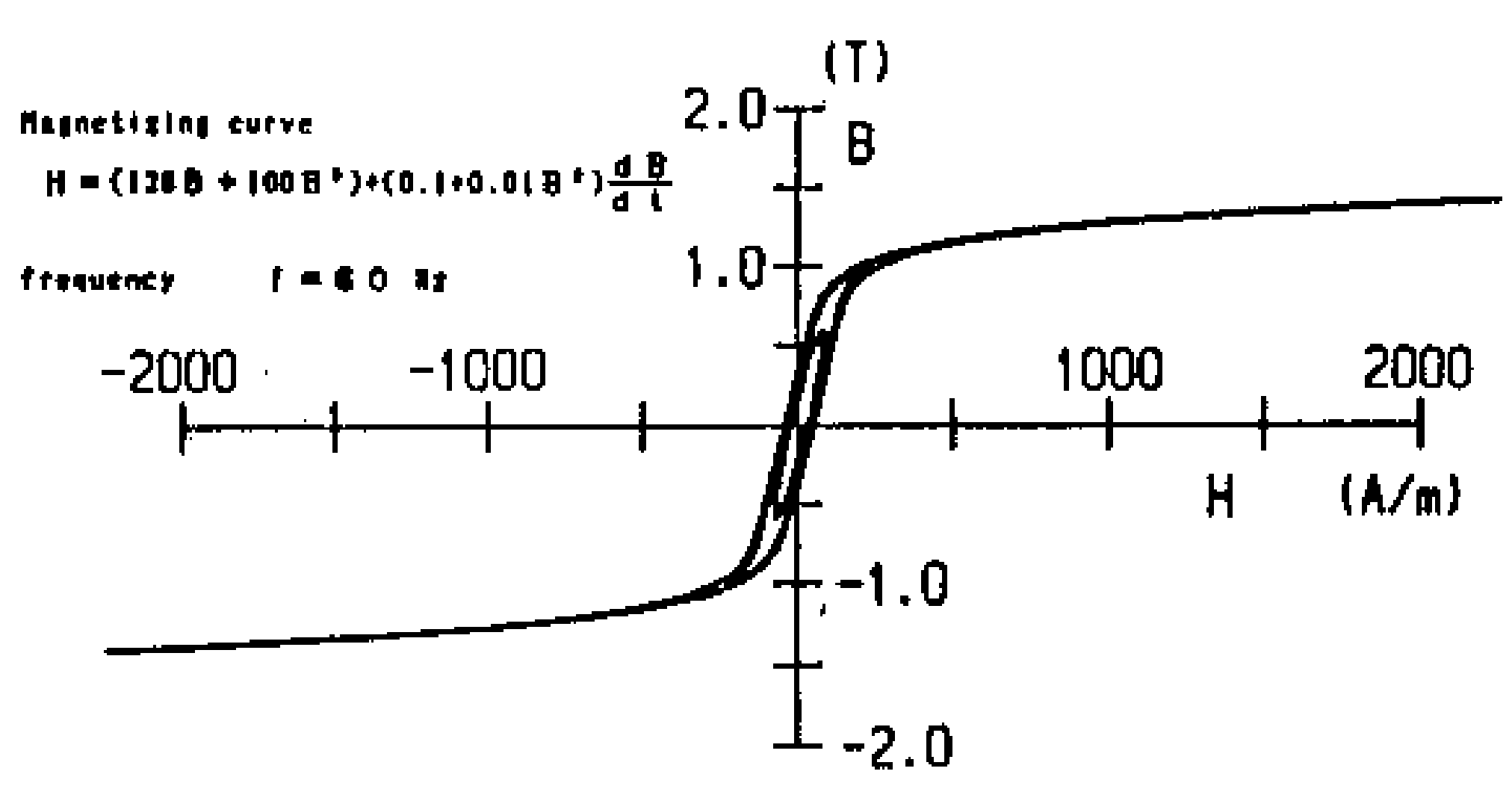

Fig. 8 B-H curve of saturated core with hysteresis
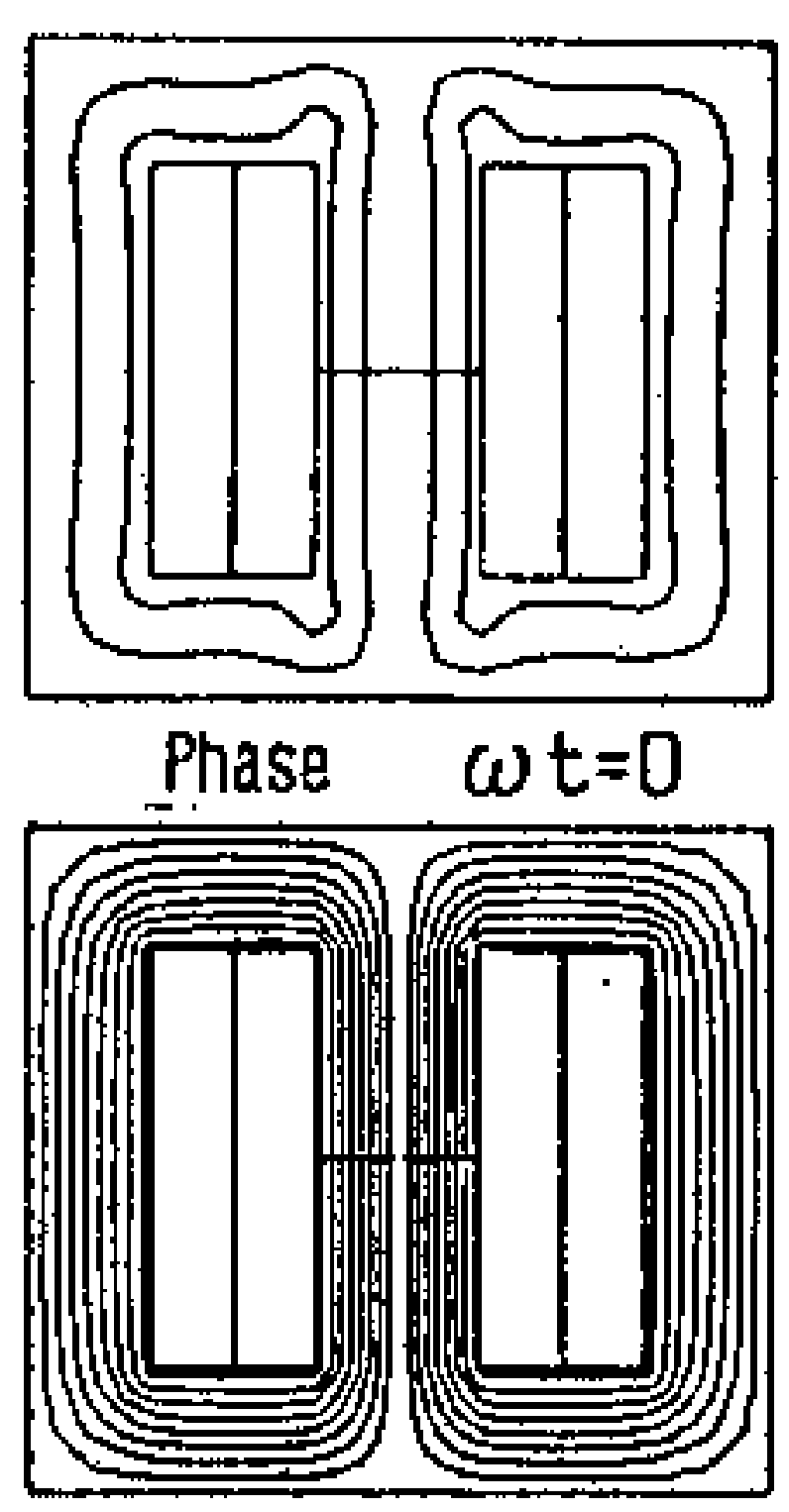

$\omega t=30$

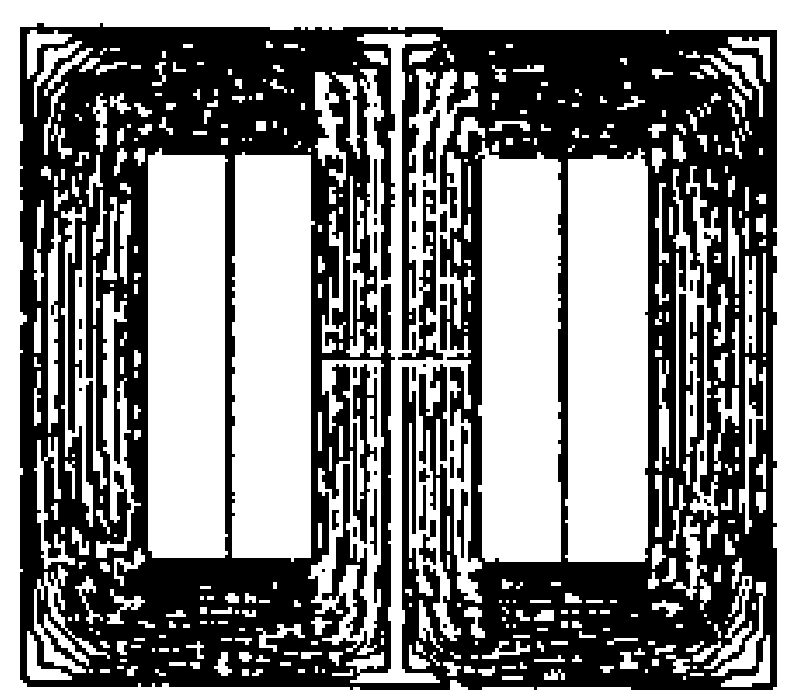

$\omega t=90$
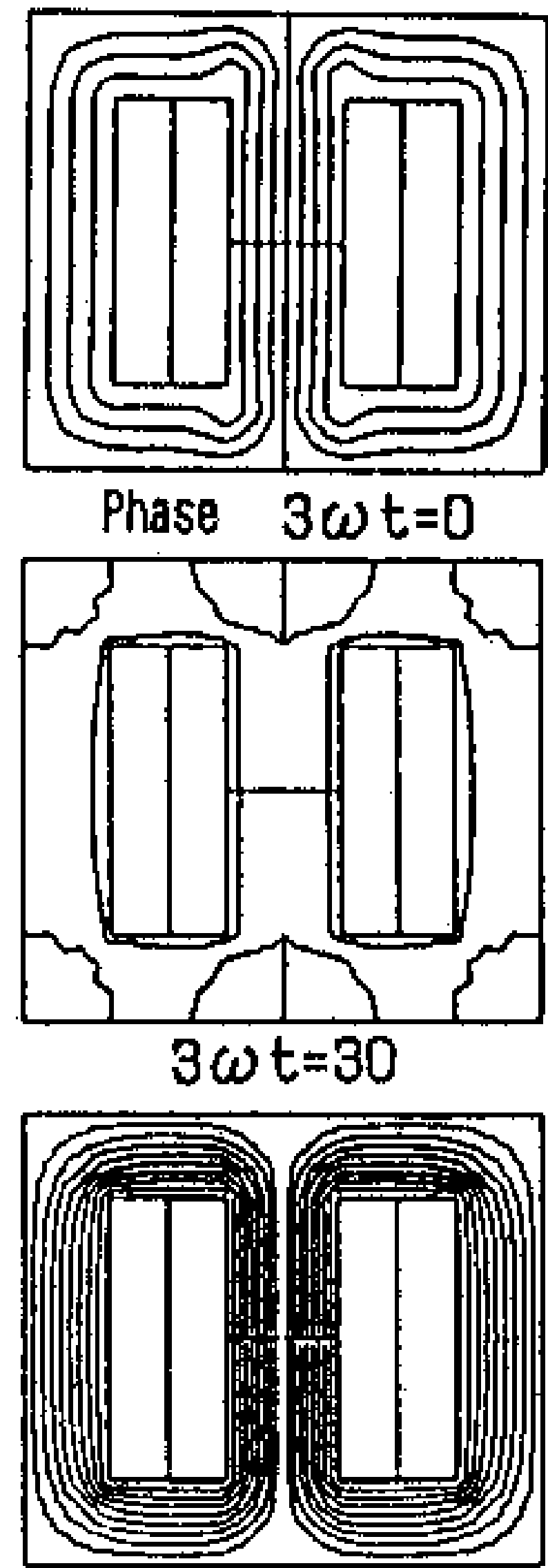

$3 \omega t=90$
Fig.9 Flux distributions to each harmonic component

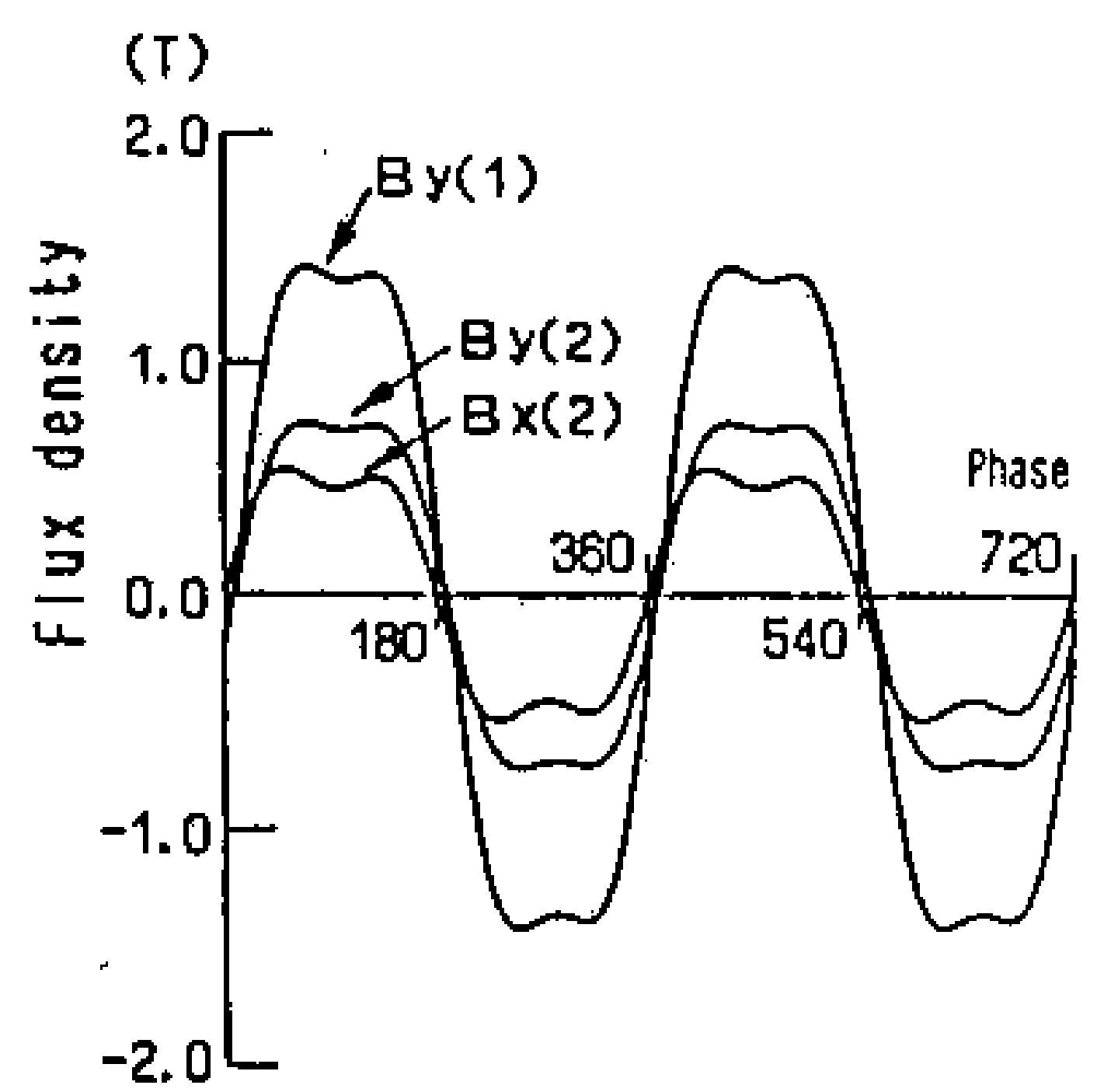

Fig. 10 Waveform of flux density

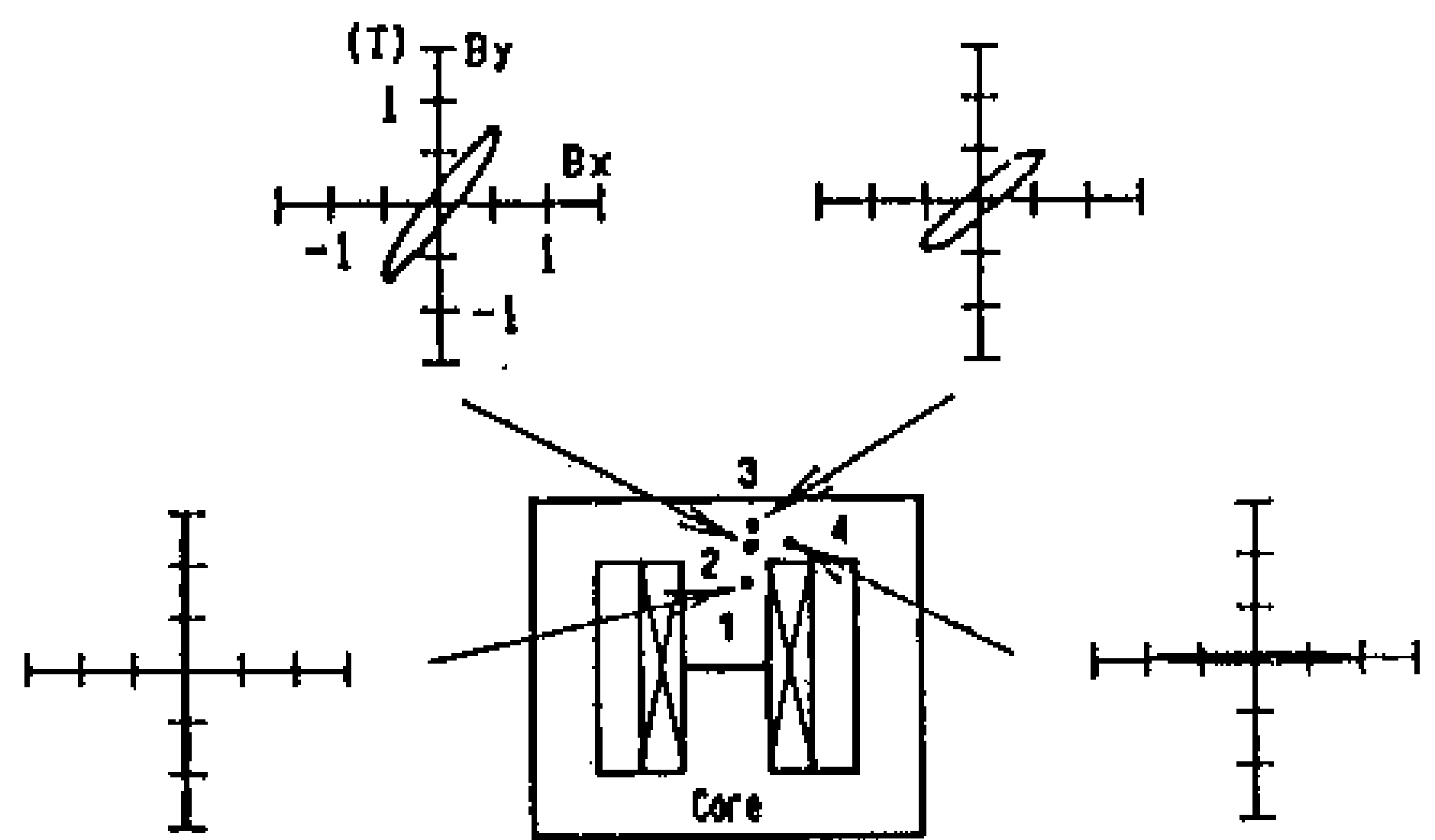

Fig.11 Vector loci of rotating field

\section{Conclusions}

From the above analysis of time-periodi magnetic fleld with saturation and hysteres characteristics the conclusions can be drawn The compartsons between numerica analysis and experimental results show reasonable agreement.

It $1 \mathrm{~s}$ efficient to use HBFEM fo designing Tripler and some special hybr high speed motor connected with power sourc and external circuit, in which the thir harmonic components play an important role.

It should be pointed that the analys considered with hysteresis characteristi needs further investigation.

\section{References}

[1] S.Yamada, K.Bessho : "Harmontc Fiel" Calculation by the Combination o: Finite Element Analysis and Harmonir Balance Method" " Magnetics, $\frac{\text { IEEE }}{\text { Vol. Trans. }}$ pp.2588-2590, 1988 .

[2] J.G.Santesmases etc.: "Analytical Approximation of Dynamic Hysteresis Loop and Its Application to a Serie? Ferroresonant circult" Proc. IEE Vol.117, No.1 pp. 234-240, 1970

(3) S.Yamada, K.Bessho, J.Lu : "Harmonic Balance Finite Element Method $t$ Applied Nonlinear AC Magnetic Analysis", IEEE Trans. Magnetics, Vol.MAG-25, No.4, pp.22971- $29 \overline{3}, \overline{9} \overline{8} \mathrm{c}$ 